\title{
The relation between overall noisiness and instantaneous judgment of noise and the effect of background noise level on noisiness
}

\author{
Seiichiro Namba and Sonoko Kuwano \\ College of General Education, Osaka University, \\ 1-1 Machikaneyama-cho, Toyonaka, Osaka, 560 Japan
}

(Received 1 May 1979)

\begin{abstract}
In order to evaluate community noise, the measurement of long-term noisiness is very important. However the conventional psychophysical method is inappropriate for this purpose. Using the newly developed "method of continuous judgment by category," the relationship between instantaneous judgment and long-term noisiness was examined. Aircraft noises overlapping background noises were adopted as stimuli. As a result, it was suggested that long-term noisiness was determined by the prominent parts of instantaneous judgment. And it was made clear that the aircraft noise to background noise ratio had much effect on the overall noisiness of aircraft noises.
\end{abstract}

PACS number: 43. 50. Ba

\section{INTRODUCTION}

It is important to evaluate the long-term noisiness of environmental noise, but very difficult to measure it in experimental situations. The long-term noisiness could be measured by a social survey, but a social survey is usually intended to research many kinds of social and personal factors which would affect the noisiness of noise as well as the noisiness itself, and is not always appropriate to make clear the relationship between the noisiness and the physical properties.

Concerning environmental noise, whose level is fluctuating at each moment, we have two kinds of impressions; those are instantaneous noisiness corresponding to the level fluctuation at each moment and overall (long-term) noisiness. As the long-term noisiness of such level-fluctuating noise is assumed to be a kind of summation or the average of instantaneous noisiness, the long-term noisiness could be predicted from instantaneous judgments, which are thought to have good correspondence with physical properties of sound. In order to measure the instantaneous judgment of noise, we have developed a new method called "the method of continuous judgment by category)". This method has the advantages that it may be applied to stimuli of longer durations and it allows subjects to pay no attention to any specific stimuli such as aircraft noise.

To determine the relationship between instantaneous judgments and the long-term effects of noise, it is necessary to measure both of them using the same stimulus. And the stimulus should have an appropriate duration whose overall noisiness and instantaneous judgment can be easily judged.

Aircraft noises overlapping background noises were adopted as stimuli. Using these stimuli we could also examine the effect of the level of background noises on the judgment of the stimuli. This effect is supposed to be a very important factor of long-term noisiness ${ }^{2-4}$. The durations of the stimuli were about 10 minutes. Moreover by giving a longer period between presentation of stimuli and judgment, the noisiness of stimuli of longer durations $(60 \mathrm{~min}$ or $120 \mathrm{~min})$ were also examined. 


\section{EXPERIMENT}

In the experiment, the overall noisiness of 10-, 60- and 120-minutes stimuli were obtained and their relation with instantaneous judgment were examined. The effect of the level of background noise (abbreviated $\mathrm{BN}$ ) on the noisiness of aircraft noise (abbreviated AN) was also tested.

\subsection{Subjects}

Twelve female subjects aged 20 to 40 years with normal hearing ability participated in the experiment. They were all housewives and, except for one subject, did not have any experience with psychological experiments before.

\subsection{Stimuli and Apparatus}

a) Five peak levels of ANs were used; 55, 60, 65, 70 and $75 \mathrm{~dB}(\mathrm{~A})$. The noises were derived from a Boeing 747, Douglas DC-10, Lockheed L-1011, and Boeing 727. They were flying mode $-7.5 \mathrm{~km}$ from the end of runway B of Osaka International Airport. The durations of the ANs were about 40 seconds. b) BNs were recorded on magnetic tapes at the center of a field and the side of a four-lane road in Osaka Prefecture. It included various kinds of distant sounds such as road traffic noise, birds twittering, human voices. The durations of the BNs were about 10 minutes. c) $\mathrm{AN}$ and $\mathrm{BN}$ were electrically mixed by a mixer (SONY MX710) and presented to each subject in a sound proof room through an amplifier (YAMAHA CA1000III) and a loudspeaker (DIATONE DS301). The sound levels of $\mathrm{AN}$ and $\mathrm{BN}$ are shown in Table 1 and examples of the stimulus patterns are shown in Fig. 1. Leq of the ANs were calculated from the sound level measured every $500 \mathrm{~ms}$. The peak level of $\mathrm{AN}$ is the maximum value during the AN presentation to the subjects. $L e q, L_{10}$ and $L_{50}$ of the BNs were calculated from the sound level measured every $5 \mathrm{~s}$.

\subsection{Procedure}

There were two sessions in the experiment. In the one session only $\mathrm{BN}$ was presented (condition $\mathrm{AN}-$ ), and in the other session electrically mixed $\mathrm{AN}$ and $\mathrm{BN}$ were presented (condition $\mathrm{AN}+$ ). In the $\mathrm{AN}+$ condition, the interval between ANs was about 2 minutes which was supposed to be a minimum interval of the flight schedule. Half of the subjects (6 subjects) did the AN + condition first and the other half did the $\mathrm{AN}$ - condition first.

In each session six kinds of BNs were each presented at random. In order to obtain the instantaneous noisiness, the method of continuous judgment by category was adopted. The subjects judged the noisiness of the sound of each moment using seven categories from "intolerably noisy" to "not noisy at all" by touching one of seven microswitches on a response box corresponding to each category. The responses of the subjects were con-

Table 1 Sound levels of aircraft and background noises.

(dBA)

\begin{tabular}{|c|c|c|c|c|c|c|c|c|c|}
\hline \multirow{3}{*}{ No. } & \multicolumn{3}{|c|}{ Background noise } & \multirow{2}{*}{\multicolumn{5}{|c|}{$\begin{array}{l}\text { Aircraft noise } \\
\text { Peak level }\end{array}$}} & \multirow{3}{*}{$\begin{array}{c}\mathrm{AN}+\mathrm{BN} \\
L e q\end{array}$} \\
\hline & \multirow[t]{2}{*}{ Leq } & \multirow{2}{*}{$L_{10}$} & \multirow[t]{2}{*}{$L_{50}$} & & & & & & \\
\hline & & & & TR & $\begin{array}{l}\text { DC10 } \\
\text { B747 }\end{array}$ & TR & $\begin{array}{l}\text { DC10 } \\
\text { B747 }\end{array}$ & B727 & \\
\hline 1 & 45.7 & 47.7 & 42.7 & 55 & 60 & 65 & 70 & 75 & 57.6 \\
\hline 2 & 53.4 & 54.3 & 50.5 & 55 & 60 & 65 & 70 & 75 & 58.8 \\
\hline 3 & 58.7 & 61.9 & 55.9 & 55 & 60 & 65 & 70 & 75 & 62.6 \\
\hline 4 & 35.7 & 37.7 & 32.7 & 55 & 60 & 65 & 70 & 75 & 57.3 \\
\hline 5 & 43.4 & 44.3 & 40.5 & 55 & 60 & 65 & 70 & 75 & 57.6 \\
\hline 6 & 48.7 & 51.9 & 45.9 & 55 & 60 & 65 & 70 & 75 & 60.6 \\
\hline
\end{tabular}

* No. 4-No. 6 are the stimuli, the levels of which are lowered by $10 \mathrm{~dB}$ from the levels of the stimuli No. 1 - No. 3 respectively.

** The peak levels of aircraft noises presented to the subjects were a little higher $(1.2-2.3 \mathrm{~dB})$ than the values in this table. Since the durations of the peaks were short, they are shown in $5 \mathrm{~dB}$ steps. 


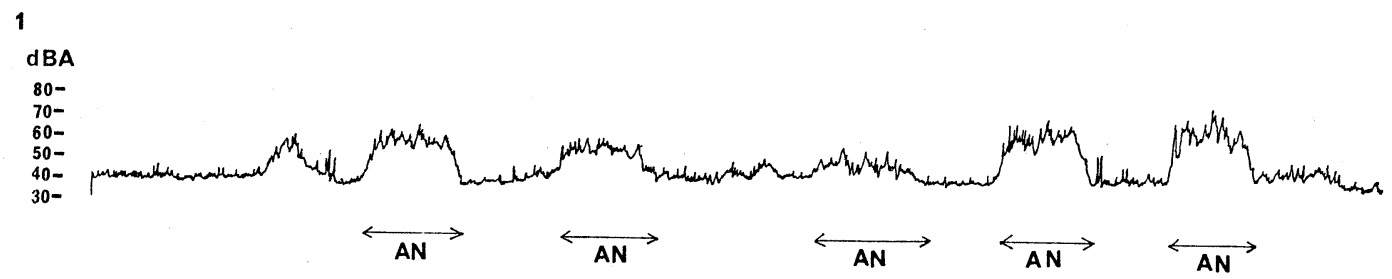

2

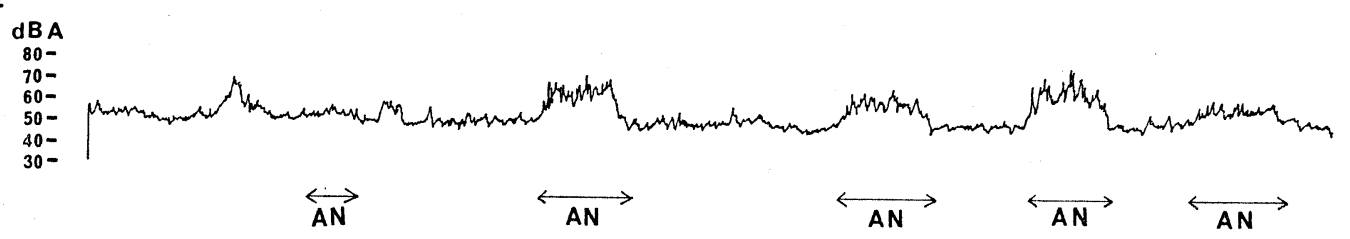

3

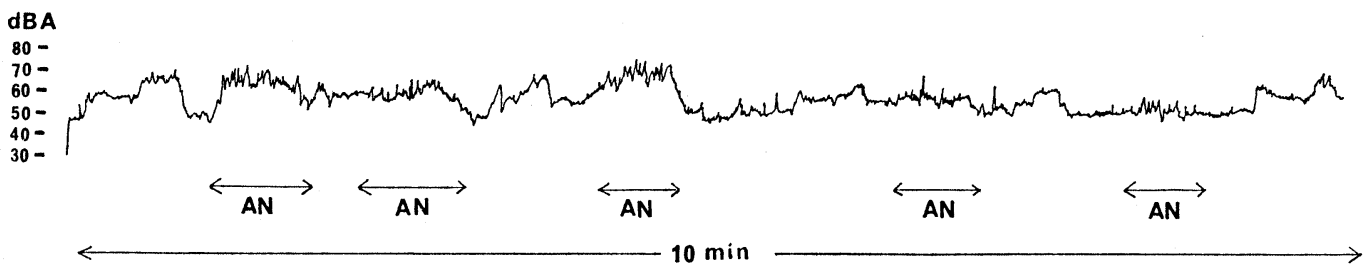

Fig. 1 Examples of the sound level patterns of the stimuli used in the experiment.

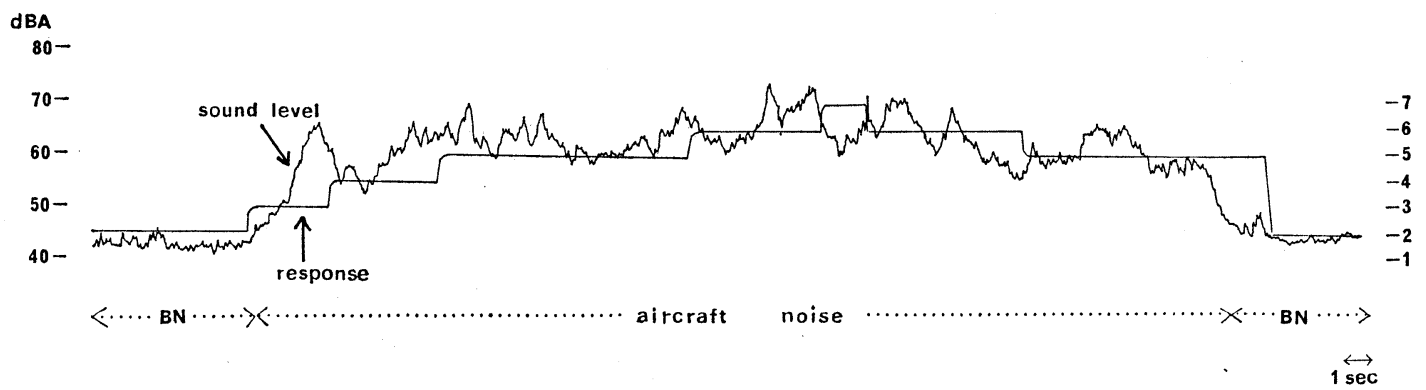

Fig. 2 An example of the record.

tinuously recorded on a level recorder (B\&K 2305). An example of the record is shown in Fig. 2. After the instantaneous judgment of each 10minutes stimulus was finished, the subjects were asked to fill out a questionnaire, in which they were surveyed concerning the overall noisiness of the 10-minutes noise using the same seven categories used in the instantaneous judgment, the difficulty of the judgment, degree of fatigue, sound sources, the noisiest sound, the loudest sound, the most prominent sound, and the subjective duration of the experiment.
About one month after the experiment, we called each subject and asked her to judge the overall noisiness of each session, $\mathrm{AN}+(60 \mathrm{~min}), \mathrm{AN}-$ $(60 \mathrm{~min})$, and both sessions together $(120 \mathrm{~min})$. They easily judged this long-term noisiness. The reason we called after an interval of one month is that time is needed for averaging or grasping the impression of long-term noisiness. This period between presentation of stimuli and judgment was useful to avoid the overestimation of the latter parts of the stimulus series. 


\section{RESULTS AND DISCUSSION}

1) Ten-minutes overall noisiness $\left(\mathrm{OA}_{10}\right)$ of $\mathrm{AN}-$ condition is highly correlated with $\operatorname{Leq}(r=.977$, Fig. 3), $L_{10}(r=.975)$ and $L_{50}(r=.976)$. Therefore any measures are appropriate to evaluate the overall noisiness of $\mathrm{BN}$. However in order to add the energy of $\mathrm{AN}$ and $\mathrm{BN}$, energy based Leq is very convenient. Moreover Leq usually shows high correlation with subjective responses to noise ${ }^{5-8)}$. Therefore we would like to use $L e q$ as an index in further discussion. Leq of $\mathrm{AN}+\mathrm{BN}$ ( $L e q$ of $\mathrm{AN}+$ condition) shown in the right column of Table 1 was calculated by adding the energy of $\mathrm{AN}$ and $\mathrm{BN}$. The increase of Leq caused by

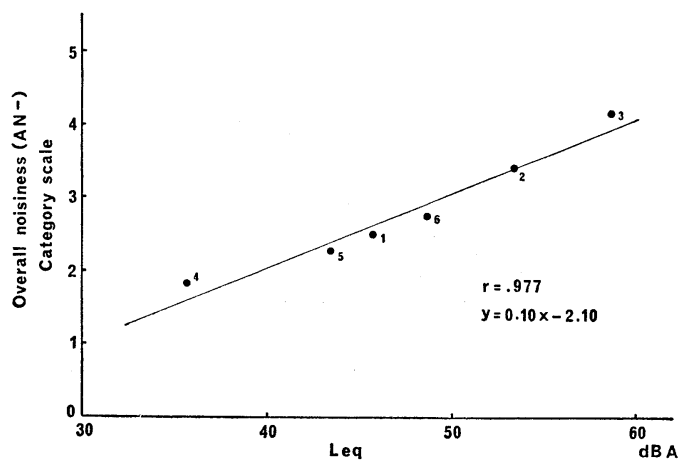

Fig. 3 Relation between 10-minutes overall noisiness $\left(\mathrm{OA}_{10}\right)$ and $L e q$ in $\mathrm{AN}-$ condition.

High correlation can be noticed between them. the overlapping of $\mathrm{AN}$ is larger when the level of $\mathrm{BN}$ (Leq of $\mathrm{AN}-$ condition) is lower. Table 2 shows the coefficient of correlation between Leq and subjective judgments $\left(\mathrm{OA}_{10}\right.$, the average of instantaneous judgment: $\overline{\mathrm{IJ}}$, the difficulty of the judgment, etc.). The relation between Leq and $\mathrm{OA}_{10}$ in $\mathrm{AN}+$ condition is shown in Fig. 4.

2) From the answers to the questionnaire the average scores of the difficulty and fatigue judgments showed that the subjects did not have difficulty or fatigue judging the instantaneous noisiness.

3) The category difference between $\mathrm{OA}_{10}$ of $\mathrm{AN}+$ and $\mathrm{OA}_{10}$ of $\mathrm{AN}$ - was used as an index of the increase of the noisiness by the overlapping of aircraft noise on environmental noise. This category difference showed high negative correlation with Leq of $\mathrm{BN}$ (Fig. 5). This fact suggests that the increase of the noisiness caused by the overlapping of $\mathrm{AN}$ is affected by the level of $\mathrm{BN}$. When the

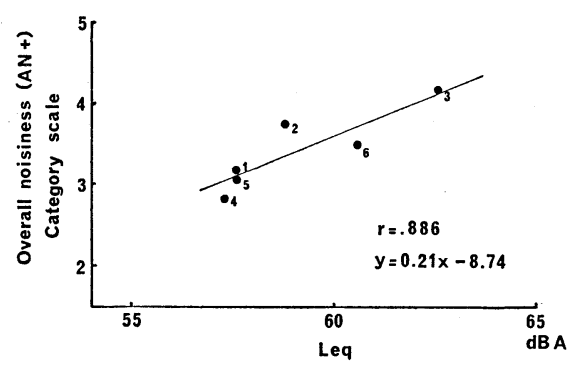

Fig. 4 Relation between 10-minutes overall noisiness $\left(\mathrm{OA}_{10}\right)$ and $L e q$ in $\mathrm{AN}+$ condition.

Table 2-a Coefficient of correlation between Leq and subjective judgments.

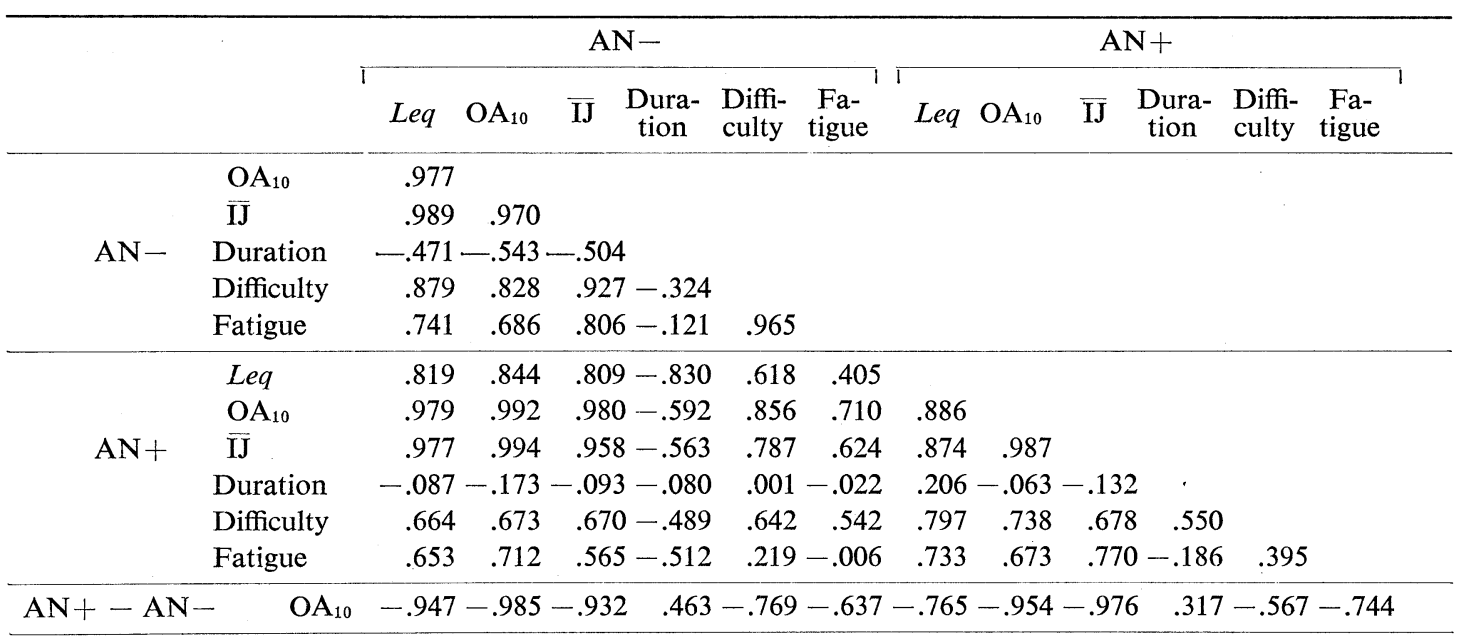


Table 2-b Coefficient of correlation between Leq and subjective judgements.

\begin{tabular}{crrr}
\hline & $\begin{array}{c}\mathrm{OA}_{60} \\
\& \\
\mathrm{OA}_{120}\end{array}$ & $\overline{\mathrm{OA}_{10}}$ & $\overline{\mathrm{IJ}}$ \\
\hline Leq & .996 & .979 & .979 \\
$\mathrm{OA}_{60} \& \mathrm{OA}_{120}$ & .993 & .993 & \\
$\mathrm{OA}_{10}$ & 1.000 & & \\
\hline
\end{tabular}

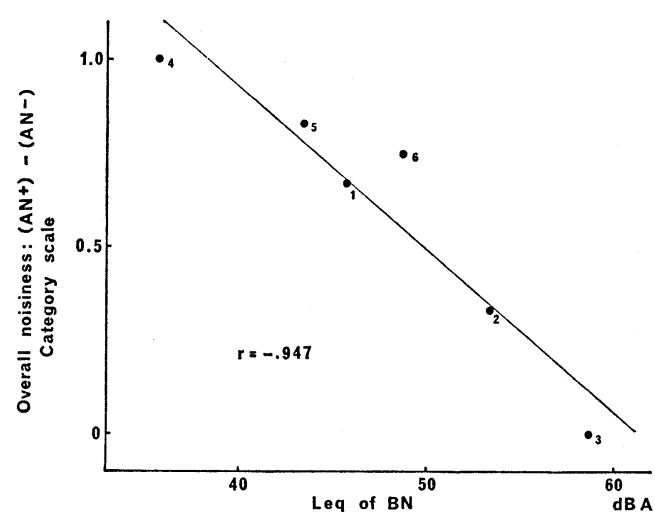

Fig. 5 Category difference between 10minutes overall noisiness of $\mathrm{AN}+$ and $\mathrm{AN}$ - conditions is plotted against Leq of background noise.

High negative correlation suggests that the effect of aircraft noises decreases as the level of background noise increases.

level of BN increased, the difference of the noisiness between $\mathrm{AN}+$ and $\mathrm{AN}-$ conditions decreased. For example, when Leq of $\mathrm{BN}$ was about $60 \mathrm{~dB}(\mathrm{~A})$, the category difference became zero. It means that under a noisy environment the flyover of aircraft is not of much effect.

4) In the position overlapping $\mathrm{AN}$ on $\mathrm{BN}$, the average of instantaneous judgment (IJ of $\mathrm{AN}+$ ) and Leq (Leq of $\mathrm{AN}+)$ were calculated. And in the corresponding parts of $\mathrm{AN}$ - condition, the average of instantaneous judgment (IJ of $\mathrm{AN}-$ ) and Leq (Leq of $\mathrm{AN}-$ ) were also calculated. These averages of instantaneous judgment were calculated giving the weight to each category corresponding to the percentage of time during which the category was used. The category difference between IJ of $\mathrm{AN}+$ and $\mathrm{IJ}$ of $\mathrm{AN}-$ (abbreviated "IJ of $\mathrm{AN}+$ - IJ of AN-") was used as an index of the increase of the noisiness by the overlapping of each aircraft noise on the background noise. Leq of $\mathrm{AN}+$ to Leq of $\mathrm{AN}$ - ratio is a kind of signal

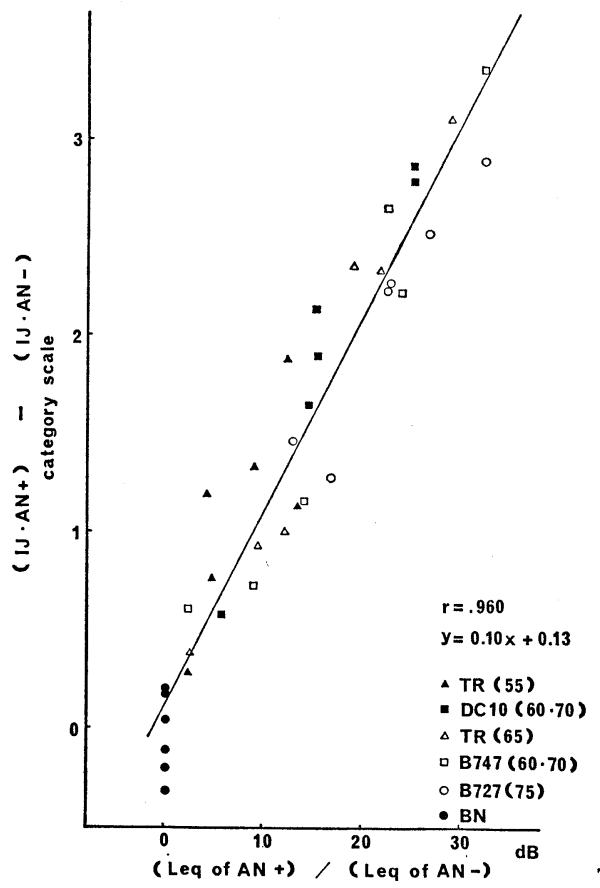

Fig. 6 Category difference between the average of instantaneous judgment of $\mathrm{AN}+$ and $\mathrm{AN}$ - conditions (" $\mathrm{IJ}$ of $\mathrm{AN}+$ - IJ of AN-") showed high correlation with Leq of $\mathrm{AN}+$ to $L e q$ of $\mathrm{AN}$ - ratio.

(aircraft noise) to noise (background noise) ratio, i.e. S/N. "IJ of AN+ - IJ of AN-" had high correlation with Leq of $\mathrm{AN}+$ to Leq of $\mathrm{AN}$ - ratio as shown in Fig. 6.

From this result, the increase of the noisiness by flying of each AN is affected by Leq of $\mathrm{BN}$. This same tendency can be seen in our previous experiment ${ }^{9)}$ using the ANs under the landing path which had shorter duration and higher frequency components.

5) In $\mathrm{AN}+$ condition, $\mathrm{AN}$ was chosen as the noisiest, the loudest and the most prominent sound of all. However the percentage of each judgment was different from one another. The loudest judgment of AN showed the highest percentage $(70.8 \%)$ and the most prominent judgment of AN showed the lowest percentage $(40.3 \%)$. This fact suggests that the loudest sound is not always the noisiest sound, nor the most prominent sound. The same tendency was found in the judgment of road traffic noise in $\mathrm{AN}$ - condition. 


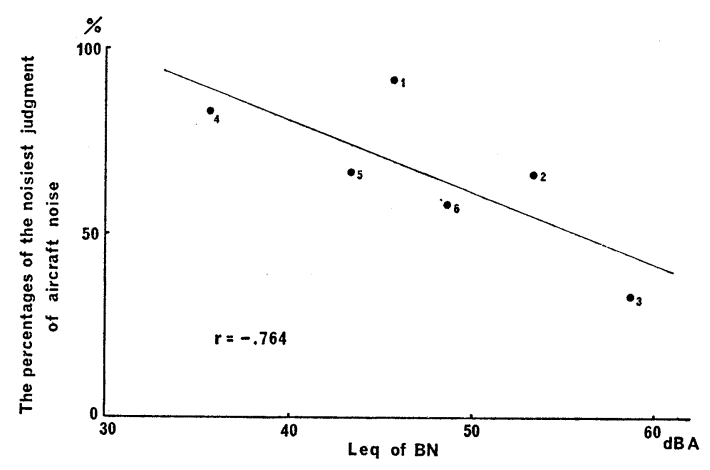

Fig. 7 Relation between the percentages of the noisiest judgment of aircraft noises and Leq of background noise.

Under a quiet background noise aircraft noises were judged very noisy, but the percentages decreased according to the increase of the level of background noise.

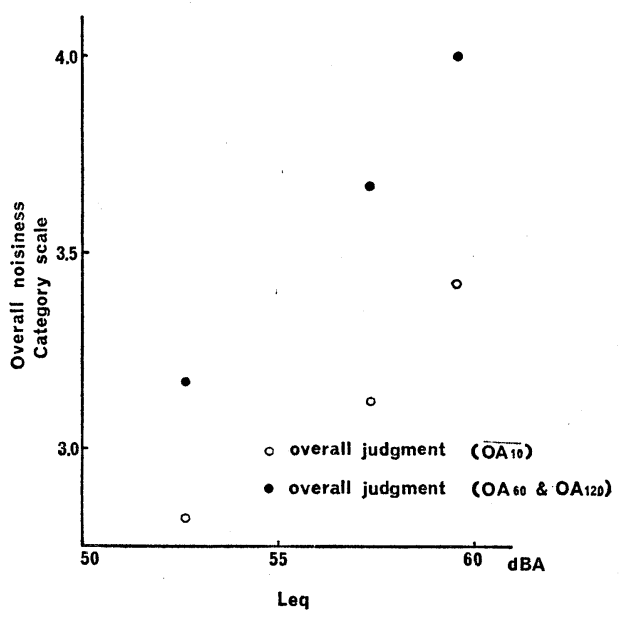

Fig. 8 10- and 60- \& 120-minutes overall noisiness are plotted against Leq. Both of them are affected by Leq.

Figure 7 shows the relation between the noisiest judgment of AN and Leq of BN. Negative correlation was found between them, i.e. the percentage of the noisiest judgment of AN decreased as Leq of $\mathrm{BN}$ increased. In fact under the noisy $\mathrm{BN}$ condition the noisiest sound was not AN, but road traffic noise.

All the results of 10-minutes overall judgment, instantaneous judgment and the noisiest judgment confirmed the effect of the level of BN on the noisiness of AN.
6) In the experiment, the relation between overall noisiness and instantaneous judgment was examined using three kinds of subjective responses. They were 60- and 120-minutes overall noisiness $\left(\mathrm{OA}_{60}, \mathrm{OA}_{120}\right)$ judged one month after the experiment, the average of six or twelve 10-minutes overall noisiness $\overline{\left(\mathrm{OA}_{10}\right)}$ and the average of instantaneous judgment ( $\overline{\mathrm{IJ}})$.

The relation between $L e q$ and $\mathrm{OA}_{60} \& \mathrm{OA}_{120}$, $\overline{\mathrm{OA}_{10}}$ is shown in Table 2-b and Fig. 8, which suggests that long-term (60 min or $120 \mathrm{~min}$ ) noisiness is also affected by Leq.

7) Figure 9 shows the relation between $\mathrm{OA}_{10}$ and $\overline{\mathrm{IJ}}$. High correlation and small RMS (root mean square of the difference between both judgments) show the agreement of both judgments. This fact suggests that $\mathrm{OA}_{10}$ is determined by the average of instantaneous judgment under the condition that both stimulus duration and the interval between stimulus presentation (instantaneous judgment) and overall judgment are not so long.

8) $\mathrm{OA}_{60} \& \mathrm{OA}_{120}$ and $\overline{\mathrm{OA}_{10}}$ are plotted against $\overline{\mathrm{IJ}}$ in Fig. 10. $\mathrm{OA}_{60} \& \mathrm{OA}_{120}$ increased in proportion to $\overline{\mathrm{OA}_{10}}$ and $\overline{\mathrm{IJ}}$, but $\mathrm{OA}_{60} \& \mathrm{OA}_{120}$ was overestimated compared with the average of instantaneous judg-

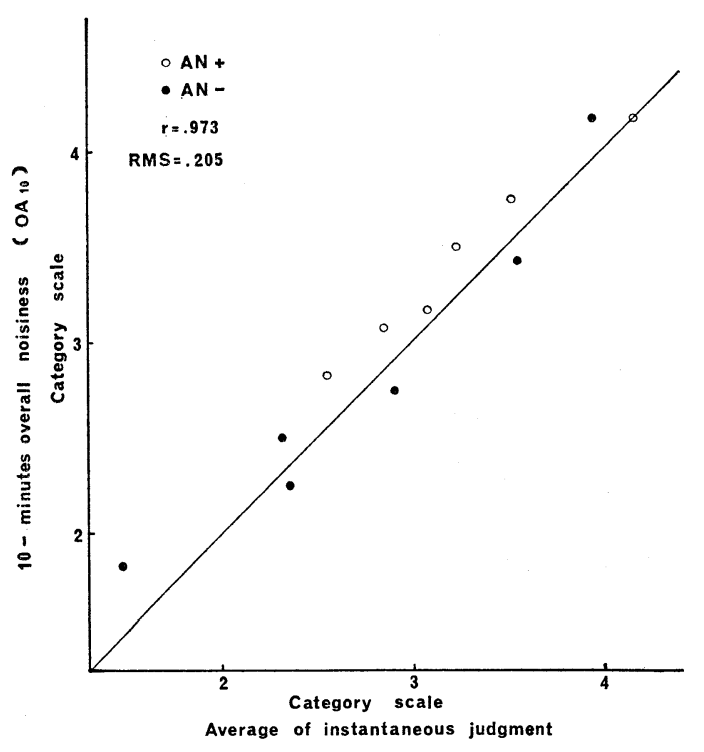

Fig. 9 Relation between 10-minutes overall noisiness and the average of instantaneous judgment.

High correlation and small RMS show the agreement of both judgments. 


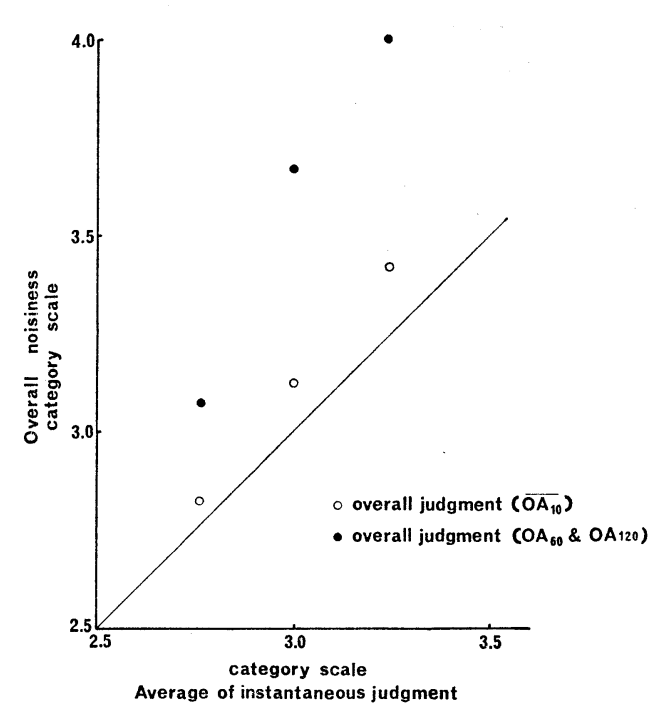

Fig. 10 10- and 60- \& 120-minutes overall noisiness are plotted against the average of instantaneous judgment.

60- \& 120-minutes overall noisiness showed overestimation compared with the average of instantaneous judgment, which suggests that 60- \& 120-minutes overall noisiness is determined by the prominent parts of instantaneous judgment.

ment. This fact suggests that the lower level parts were neglected in the judgment of long-term noisiness. And this is the same tendency as was found in our previous experiment on road traffic noise $(20 \mathrm{~min}){ }^{10)}$

\section{CONCLUSION}

1) The increase of Leq caused by the overlapping of $\mathrm{AN}$ is larger when the level of $\mathrm{BN}$ is lower. Therefore the category difference between $\mathrm{OA}_{10}$ of $\mathrm{AN}+$ and $\mathrm{OA}_{10}$ of $\mathrm{AN}$ - became larger in low $\mathrm{BN}$ conditions. This fact suggests that the effect of the overlapping of $\mathrm{AN}$ on a certain $\mathrm{BN}$ depends on the level of BN.

2) The category difference between the instantaneous judgment of $\mathrm{AN}(\mathrm{AN}+)$ and the instantaneous judgment of $\mathrm{BN}(\mathrm{AN}-)$ was highly correlated with the relation between the levels of $\mathrm{AN}$ and $\mathrm{BN}(\mathrm{S} / \mathrm{N})$. The same tendency was found in our previous experiment. ${ }^{9)}$

3) The noisiest judgment of $\mathrm{AN}$ decreased as the level of BN increased. The noisiest sound was
$\mathrm{AN}$ under the quiet $\mathrm{BN}$ condition, but it was road traffic noise under the noisy $\mathrm{BN}$ condition.

4) The long-term noisiness was found to increase in proportion to the increase of $L e q$, but it was overestimated compared with the average of instantaneous judgment.

From these findings it was suggested that the long-term noisiness is not an average of instantaneous judgment, but determined by the prominent parts of instantaneous judgment, and that the increase of noisiness by the overlapping of AN on $\mathrm{BN}$ is dependent on the relation between the levels of $\mathrm{AN}$ and $\mathrm{BN}$, i.e. $\mathrm{S} / \mathrm{N}$. Under the high $\mathrm{BN}$ condition the noisiness does not increase even when $\mathrm{AN}$ is overlapped on $\mathrm{BN}$, and under the low $\mathrm{BN}$ condition the noisiness is entirely dependent on AN.

\section{ACKNOWLEDGEMENT}

A part of this study was supported by a Grant in Aid for Scientific Research ( $\$ 345017)$, Ministry of Education.

\section{REFERENCES}

1) S. Namba, S. Kuwano, and T. Nakamura, "Rating of road traffic noise using the method of continuous judgment by category," J. Acoust. Soc. Jpn. 34, 29-34 (1978) (in Japanese).

2) C. A. Powell and C. G. Rice, "Judgments of aircraft noise in a traffic noise background," J. Sound Vib. 38, 39-50 (1975).

3) K. S. Pearsons, "The effects of duration and background noise level on perceived noisiness," FAA, FA65WA-1180 (1966).

4) D. C. Nagel, J. E. Parnell, and H. J. Parry, "Procedure for correcting perceived noise level calculations for the effect of background noise," in Transportation noise, J. D. Chalupnik, ed. (Ann Arbor Science, Michigan, 1970), pp. 187-196.

5) S. Namba, T. Nakamura, and S. Yasuda, "The relation between the loudness and the mean of energy of level-fluctuating noises," Jpn. J. Psychol. 43, 251-260 (1972) (in Japanese).

6) S. Namba, S. Kuwano, and T. Kato, "An investigation of $L e q$ and $L \alpha$ in relation to loudness," J. Acoust. Soc. Jpn. 34, 301-307 (1978) (in Japanese).

7) S. Namba, S. Kuwano, and T. Kato,"An investigation of $L e q, L_{10}$ and $L_{50}$ in relation to loudness," ASA, ASJ Joint Meeting (1978).

8) K. Hiramatsu, H. Wakasa, K. Takagi, and T. Yamamoto, "Annoyance of fluctuating noise (the validity of Leq)," J. Acoust. Soc. Jpn. 34, 641-649 (1978) (in Japanese). 
9) S. Namba and S. Kuwano, "On the noisiness of various sounds overlapping one another," Reports of the 1978 Autumn Meeting of ASJ (1978), pp. 443-444 (in Japanese).
10) S. Kuwano and S. Namba, "On the loudness of road traffic noise of longer duration $(20 \mathrm{~min})$ in relation to instantaneous judgment," ASA, ASJ Joint Meeting (1978). 